\title{
Evolution of Surgical Resection for Duodenal Duplication Cyst
}

\author{
Kevin L. Anderson, Jr, BS, Kristy L. Rialon, MD, Theodore N. Pappas, MD, Alexander Perez, MD \\ School of Medicine (Mr Anderson) and Department of Surgery (Drs Pappas and Perez), Duke University Medical Center, \\ Duke University, Durham, North Carolina, USA. \\ Department of General and Thoracic Surgery, The Hospital for Sick Children, Toronto, Ontario, Canada (Dr. Rialon).
}

\begin{abstract}
Introduction: Duodenal duplication cysts are rare congenital malformations that are most often identified incidentally and managed nonoperatively. Because of the location of the cyst, it can obstruct the biliopancreatic ampulla requiring operative intervention. An open surgical approach is the conventional procedure for these patients.

Case Descriptions: Since 1990, our institution has resected 3 duodenal duplication cysts. Symptomatic 15- and 17-year-olds underwent open resection of duplication cysts. Most recently, a 25-year-old woman presented with recurring pancreatitis and was subsequently diagnosed with a duodenal cyst on computed tomographic scan and endoscopic ultrasonography. The patient was taken to the operating room for laparoscopic excision. Operative time for the laparoscopic approach was 2 hours and 45 minutes, with no significant intraoperative blood loss. Postoperative recovery was uneventful in all cases.
\end{abstract}

Discussion: After identification of appropriate operative candidates, laparoscopic resection for duodenal duplication cysts is a feasible procedure that provides smaller incisions and decreased blood loss.

Key Words: Duodenal duplication cyst, Laparoscopic resection, Laparoscopy, Surgical treatment.

\footnotetext{
Citation Anderson K, Rialon K, Pappas T, Perez A. Evolution of surgical resection for duodenal duplication cyst. CRSLS e2016.000108. DOI: 10.4293/CRSLS.2016.000108.

Copyright (c) 2017 by SLS, Society of Laparoendoscopic Surgeons. This is an open-access article distributed under the terms of the Creative Commons Attribution-Noncommercial-ShareAlike 3.0 Unported license, which permits unrestricted noncommercial use, distribution, and reproduction in any medium, provided the original author and source are credited.

This research was supported by a National Institutes of Health TL-1 Clinical and Translational Science Award (CTSA), Grant 1UL1-TR001117-01 from the National Center for Advancing Translational Sciences (NCATS).

Disclosures: Dr. Pappas receives fees from Transenterix Corporation, makers of a surgical robot. The remaining authors report no disclosures.

Address correspondence to: Kevin L. Anderson, Jr, BS, School of Medicine, Duke University, Duke University Medical Center, 7672 HAFS, Box 3448 DUMC, Durham, NC 27710. Telephone: 502-457-7753, Fax: 919-681-7934, E-mail: kla24@duke.edu
}

\section{INTRODUCTION}

A gastrointestinal (GI) duplication cyst is a rare congenital malformation that can present in both children and adults. It is physically described as a spherical mass surrounded by a muscular coat and lined by a mucous membrane. These GI tract cysts can be categorized as foregut, small bowel, or large bowel duplications, depending on their location. In the general population, the collective estimated prevalence of these cysts ranges from 1:4,500 to 1:10,000 with most cases occurring in children. ${ }^{1}$ Duodenal duplication cysts represent less than $5 \%$ of all GI duplications. Whereas some patients may present clinically with a palpable mass, abdominal pain, and bleeding, most pa- tients are asymptomatic, and the cysts are discovered incidentally during diagnostic workup. ${ }^{2}$ These entities are even less common in adults, where treatment traditionally involves surgical resection when symptomatic. ${ }^{3-5}$ Duodenal duplication resection can be challenging, even for the most experienced surgeons because of the anatomic proximity of these cysts to the ampulla.

Extraluminal intestinal duplication cysts are most commonly treated with open exploration and resection, but a few reports have detailed the use of laparoscopic resection of gastric and ileal duplication cysts in children and adults. ${ }^{6-9}$ After detailing 2 cases of open surgical resection of duodenal duplication cysts, we report one of the few known cases of a laparoscopic resection of a duodenal 
duplication cyst in an adult. With the proven perioperative benefits of minimally invasive surgery, such as faster recovery and improved perioperative outcomes, it is critical that surgeons feel comfortable in identifying operative candidates for laparoscopic resection and are comfortable with the technique needed to provide the optimal outcome. ${ }^{10,11}$

\section{CASE DESCRIPTIONS}

\section{Case 1}

A 15-year-old female was referred to our institution for evaluation of recurrent pancreatitis. She underwent endoscopic retrograde cholangiopancreatogram (ERCP), which demonstrated both a duodenal duplication and incomplete pancreatic divisum. The patient was then scheduled for a resection of the duodenal duplication with a sphincterotomy of both major and minor papillae.

After induction of general anesthesia, a right upper quadrant incision was made, and the abdominal cavity was entered and explored. Initial inspection showed a grossly normal liver, stomach, kidneys, and large and small bowels. A Kocher maneuver was performed to visualize the duodenum, followed by palpation of the duplication cyst in the area of the major and minor papillae. A longitudinal anterior duodenotomy was then made over the duplication cyst. Upon entering the duodenum and identifying the duplication cyst, $75 \mathrm{U}$ of secretin were given intravenously to verify the location of the accessory pancreatic duct. Using electrocautery, the duplication was excised off the wall of the duodenum. Overall, the patient tolerated the procedure well. The operative time for this patient's duodenal cyst was 3 hours and 25 minutes. The patient remained in the hospital until she was discharged on postoperative day 6 . The patient has needed no further operations and has not returned to our institution with any new symptoms.

\section{Case 2}

A 17-year-old male presented to our clinic with episodic right upper quadrant abdominal pain associated with nausea and vomiting. After thorough GI evaluation, which included an abdominal x-ray and upper GI fluoroscopy without contrast, an intraluminal duodenal duplication was diagnosed, with partial obstruction of the second portion of the duodenum. Because of this patient's symptomatic presentation, it was recommended that he undergo an open surgical resection.
The procedure was performed through a right upper quadrant transverse incision. Duodenal mobilization was again performed via Kocher maneuver. The duodenum was opened directly opposite the pancreas to minimize risk of ampullary injury, and duplication was encountered immediately. Electrocautery was used for resection down to the base on the lateral duodenal wall.

The duodenum was then opened longitudinally and closed transversely, in a Heineke-Mikulicz style. A 2-layer closure was performed using inner layer Vicryl and outer layer Lembert-style sutures. The abdomen was then irrigated, and the fascia and skin were closed in multiple layers. The operative time for this procedure was 3 hours and 15 minutes. The patient remained in the hospital until postoperative day 7 and was discharged home. A year after the operation, the patient experienced no further episodes of vomiting, nausea, or abdominal pain. Of note, 3 years after the procedure, the patient returned to the emergency department for nausea. Computed tomographic (CT) scans and upper GI series noted no abnormalities.

\section{Case 3}

A 25-year-old female nurse presented initially to her primary care provider with epigastric pain. She assumed it was reflux and took calcium carbonate antacid. After being awakened by the pain yet again, she felt nausea and took oral ondansetron. The patient noticed no alleviation of her pain. The pain was most notable during inspiration. She denied fevers, chills, and vomiting and had no history of surgery, trauma, Helicobacter pylori infection, bleeding, reflux, abdominal pain, or weight loss. On physical examination, the patient had a soft, nondistended abdomen with normal bowel sounds and no palpable masses. She was tender to palpation in the epigastric area only. On routine blood tests, she had an elevated white blood cell count and lipase; otherwise, laboratory results, including H. pylori and sedimentation rate, were unremarkable.

CT imaging (Figure 1) of the abdomen and pelvis was performed with intravenous contrast. Within the second portion of the duodenum was a well-circumscribed fluidattenuation lesion measuring $4.3 \times 3.3 \mathrm{~cm}$. The pancreas, gall bladder, and pancreatic duct were all normal appearing. EUS findings showed an anechoic, subepithelial lesion measuring $49.3 \times 38.9 \mathrm{~mm}$ in diameter adjacent to and compressing the ampulla. 


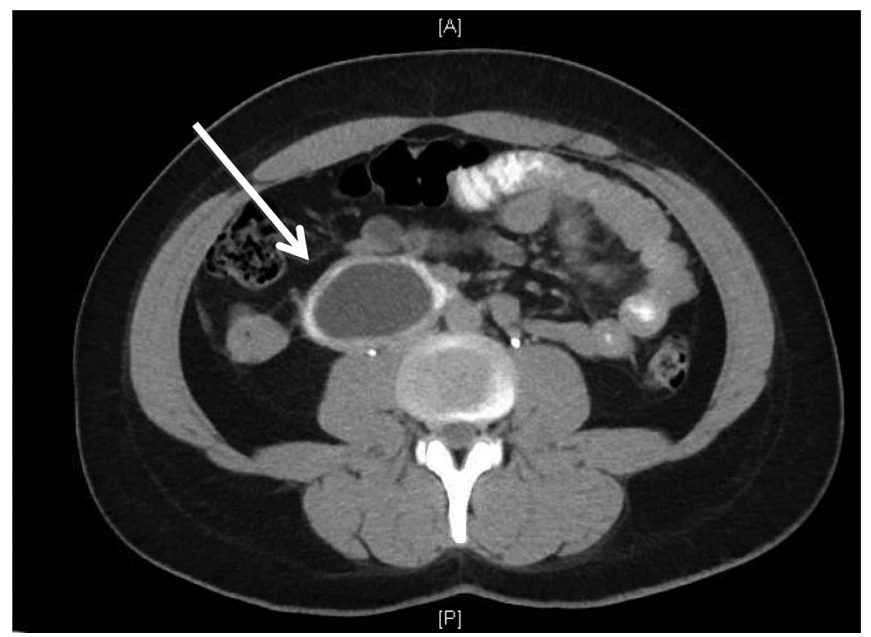

Figure 1. An abdominal CT scan demonstrating a cystic mass within the duodenum (case 3).

\section{Surgical Technique}

A Veress needle was placed in the left upper quadrant, and the abdomen was insufflated to $15 \mathrm{~mm} \mathrm{Hg}$. A 5-mm port was placed in the left upper quadrant. A $12-\mathrm{mm}$ port was placed above the umbilicus, and 3 additional $5-\mathrm{mm}$ ports were placed throughout the abdomen, including 1 for liver retraction. Anatomic attachments between the gallbladder, duodenum, and colon were taken down. A Kocher maneuver was then performed to bring down the transverse colon and medialize the duodenum. Esophagogastroduodenoscopy (EGD) was performed to localize the duplication cyst in the second part of the duodenum (Figure 2). Stay sutures were then placed around the duodenum, and it was opened longitudinally. A large cyst was noted near the ampulla, making it difficult to resect without compromising outflow from the ampulla. Using blunt dissection and a Ligasure device (Medtronic Minimally Invasive Therapies, Dublin, Ireland), along with a focus on ampulla preservation, the cyst wall was removed from the duodenal mucosa en bloc, with only a small remnant of the cyst left. The sample was then sent to pathology. The duodenotomy was closed transversely with running V-Loc and Endo Stitch sutures (Covidien; Medtronic, Minneapolis, Minnesota, USA), the abdomen was irrigated, and the stomach was insufflated via a nasogastric tube, with no bile leakage from the anastomosis. A 19 French Blake drain was placed near the anastomosis.
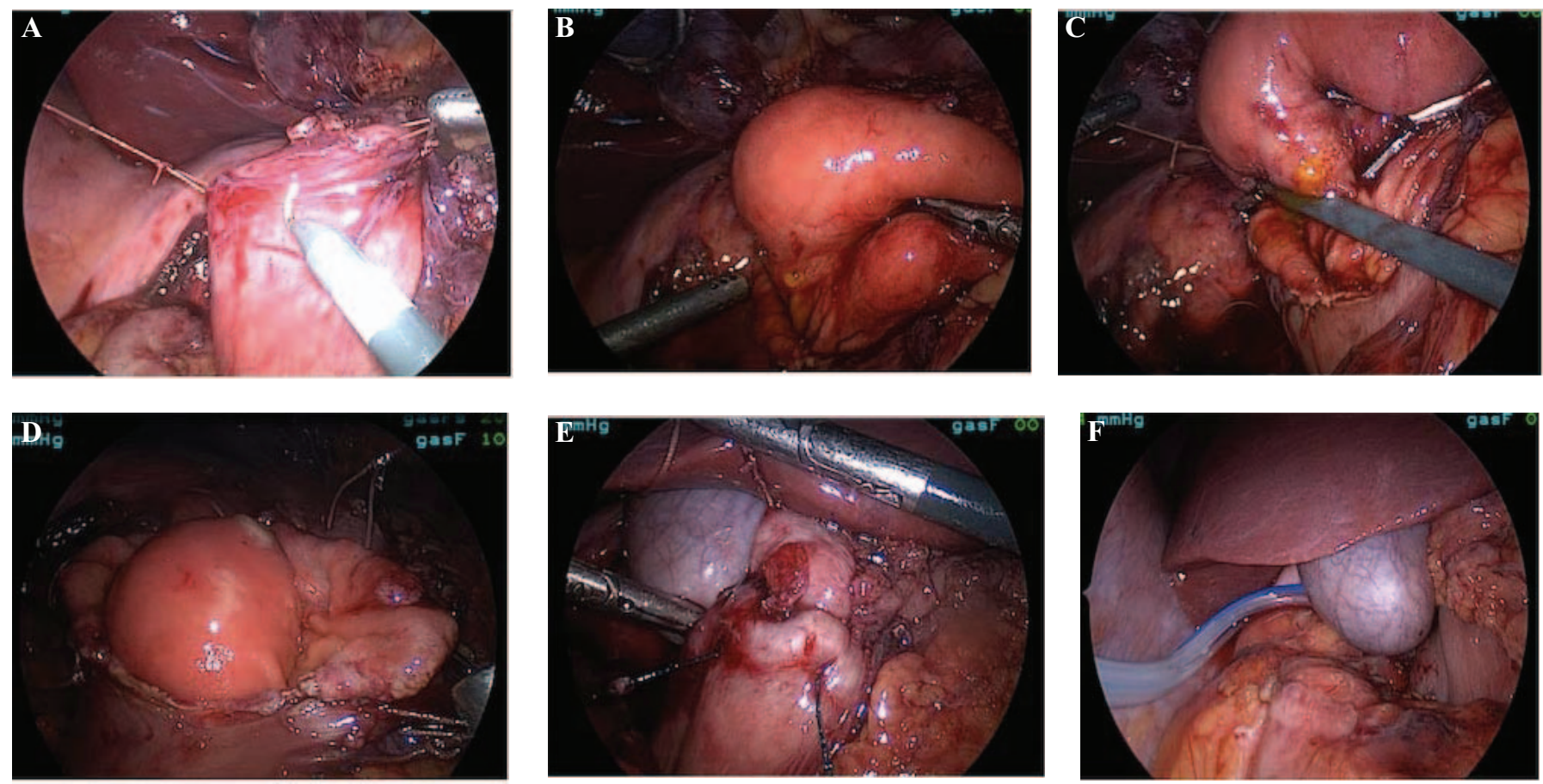

Figure 2. Intraoperative Pictures of Duodenal Duplication Cyst Resection. A: Stay sutures placed on both sides and duodenotomy made with electrocautery B: Duplication cyst visible in second portion of duodenum. C: Elevation of duplication cyst with bile seen at base. D: Duplication cyst with ampulla with bile coming from ampulla. E: Duodenum closed transversely. F: Drain placed in right upper quadrant. 
On gross examination, the pathology report described the sample as a $3 \times 2.4 \times 0.2$-cm fragment of duodenum with pink-tan mucosa on each side, which was unremarkable, with no notable lesions. The pathologic diagnosis was a duodenal duplication cyst.

Operative time was roughly 2 hours and 45 minutes with less than $50 \mathrm{~mL}$ of blood loss. On postoperative day 4 , the patient had no complaints and denied fevers, chills, abdominal pain, nausea, or vomiting. At this point, she tolerated a clear liquid diet and the amount of fluid draining was about $10 \mathrm{~mL}$ per shift. The drain was removed before discharge. At the first follow-up visit, on postoperative day 10 , she reported that she had recovered well and was tolerating a full diet.

\section{DISCUSSION}

The appropriate management of patients with duodenal duplication cysts is dependent on complications, anatomic location, and characteristics of the cyst. In a metaanalysis by Chen et $\mathrm{al}^{12}$ of 46 cases of duodenal duplication cysts, 33 (70.2\%) underwent operative management. Of those, 25 (76\%) received surgical resection, 6 (18\%) had surgical marsupializations, and 2 (6\%) underwent pancreaticoduodenectomy. In the same study, 12 of the 46 patients were treated with endoscopic marsupialization, and the remaining patient underwent medical treatment. In a case series by Antaki et al, ${ }^{1} 8$ patients with intraluminal duodenal duplication cysts had endoscopic incision and marsupialization of the cysts, with no major complications. We recognize that endoscopy is a feasible alternative to surgical resection, but it is at times limited in its ability to visualize the entire cyst and its relationship to surrounding pertinent structures, such as the ampulla. Endoscopy, compared to surgical resection, at times can result in incomplete ablation of the cyst mucosa, which has the possibility of developing malignant degeneration. ${ }^{1,13}$

Over the past 3 decades, minimally invasive surgery has matured to the point where qualified surgeons can perform most general surgical procedures laparoscopically. ${ }^{14}$ Initially, laparoscopy was used as a diagnostic procedure, but now it is the gold standard for several general surgery operations, such as cholecystectomy and distal pancreatectomy for pancreatic adenocarcinoma, because of enhanced recovery for patients, which is achieved by use of smaller incisions, decreased blood loss, and a decrease in the unavoidable stress response and postoperative ileus that follows a bowel operation. ${ }^{10,14}$ Despite these benefits, doubts persist with respect to newer and more compli- cated procedures. These feelings are reinforced oftentimes because of mixed and conflicting results in the literature. This specific sentiment was present with both laparoscopic duodenectomy and pancreaticoduodenectomy. These are now accepted and used with good outcomes in high-volume centers with advanced minimally invasive surgery faculty. ${ }^{10,15-19}$

\section{CONCLUSION}

This report highlights the evolution over the past 2 decades of surgical resection of duodenal duplication cysts, from an open approach to this application of laparoscopic excision of a duodenal duplication cyst. We have illustrated the feasibility of a minimally invasive approach to this procedure in appropriately selected patients.

\section{References:}

1. Antaki F, Tringali A, Deprez P, et al. A case series of symptomatic intraluminal duodenal duplication cysts: presentation, endoscopic therapy, and long-term outcome (with video). Gastrointest Endosc. 2008;67:163-168.

2. Tjendra Y, Lyapichev K, Henderson J, Rojas CP. Foregut duplication cyst of the stomach: a case report and review of the literature. Case Rep Pathol. 2016;2016:7318256.

3. Ackerman NB. Duodenal duplication cysts: diagnosis and operative management. Surgery. 1974;76:330-333.

4. Merrot T, Anastasescu R, Pankevych T, et al. Duodenal duplications: clinical characteristics, embryological hypotheses, histological findings, treatment. Eur J Pediatr Surg. 2006;16: $18-23$.

5. Mosca F, Stracqualursi A, Persi A, Latteri S. [Duodenal duplication: report of 2 cases in adults and review of the literature (in Italian)]. Chir Ital. 2001;53:883-891.

6. Wada S, Higashizawa T, Tamada K, et al. Endoscopic partial resection of a duodenal duplication cyst. Endoscopy. 2001;33: $808-810$.

7. Jimenez M, Cadiere GB, Dapri G, Vasilikostas G, Bruyns J, Capelluto E. Duodenal duplication cyst in an adult: first simultaneous laparoscopic and endoscopic surgery. J Laparoendosc Adv Surg Tech A. 2009;19:207-210.

8. Zheng E, Burjonrappa S. Pancreatitis because of foregut duplication cyst of the pancreas treated by laparoscopic resection. J Pediatr Surg. 2010;45:e1-e3.

9. Ballehaninna UK, Nguyen T, Burjonrappa SC. Laparoscopic resection of antenatally identified duodenal duplication cyst. JSLS. 2013;17:454-458. 
10. Kokosis G, Ceppa EP, Tyler DS, Pappas TN, Perez. A laparoscopic duodenectomy for benign nonampullary duodenal neoplasms. Surg Laparosc Endosc Percutan Tech. 2015;25:158-162.

11. Yerokun BA, Sun Z, Jeffrey Yang CF, et al. Minimally invasive versus open esophagectomy for esophageal cancer: a population-based analysis. Ann Thorac Surg. 2016;102:416-423.

12. Chen JJ, Lee HC, Yeung CY, Chan WT, Jiang CB, Sheu JC. Meta-analysis: the clinical features of the duodenal duplication cyst. J Pediatr Surg. 2010;45:1598-1606.

13. Inoue $\mathrm{M}$, Nishimura $\mathrm{O}$, Andachi $\mathrm{H}$, Koga S. Early cancer of duodenal duplication: a case report. Gastroenterol Jpn. 1979;14: 233-237.

14. Al-Taan OS, Stephenson JA, Briggs C, Pollard C, Metcalfe MS, Dennison AR. Laparoscopic pancreatic surgery: a review of present results and future prospects. HPB (Oxford). 2010;12: $239-243$.

15. Abe N, Hashimoto Y, Kawaguchi S, et al. Successful treatment of large adenoma extending close to the papilla in the duodenum by laparoscopy-assisted pancreas-sparing duodenectomy. Asian J Endosc Surg. 2016;9:52-56.

16. Benetatos N, Ammori MB, Ammori BJ. Laparoscopic pancreas-preserving total duodenectomy for familial adenomatous polyposis. Surg Laparosc Endosc Percutan Tech. 2011;21:e332e335.

17. Corcione F, Pirozzi F, Sciuto A, Galante F, Bracale U, Andreoli F. Laparoscopic pancreas-preserving subtotal duodenectomy for gastrointestinal stromal tumor. Minim Invasive Ther Allied Technol. 2013;22:187-190.

18. Menon KV, Hayden JD, Prasad KR, Verbeke CS. Total laparoscopic pancreaticoduodenectomy and reconstruction for a cholangiocarcinoma of the bile duct. J Laparoendosc Adv Surg Tech A. 2007;17:775-780.

19. Poves I, Burdio F, Alonso S, Seoane A, Grande L. Laparoscopic pancreas: sparing subtotal duodenectomy. J Pancreas. 2011;12:62-65. 